\title{
A Rare and Potentially Catastrophic Infection: Primary Intestinal Aspergillosis_-Case Report in an HIV Patient
}

\author{
Cátia Dias $(\mathbb{D}$, Filipa Duarte-Ribeiro, Sara Pipa, and Margarida Mota \\ Department of Internal Medicine, Centro Hospitalar Vila Nova de Gaia/Espinho, Espinho, Portugal \\ Correspondence should be addressed to Cátia Dias; kati.diass@gmail.com
}

Received 6 April 2018; Accepted 3 June 2018; Published 8 July 2018

Academic Editor: Raul Colodner

Copyright (c) 2018 Cátia Dias et al. This is an open access article distributed under the Creative Commons Attribution License, which permits unrestricted use, distribution, and reproduction in any medium, provided the original work is properly cited.

\begin{abstract}
Aspergillus species are ubiquitous in nature; however, infection is uncommon, except in immunocompromised or immunosuppressed hosts. We present the case of a 71-year-old woman with a history of human immunodeficiency virus infection who presented with fever, weight loss, and diarrhea, posteriorly diagnosed with intestinal aspergillosis after examination of a segmental enterectomy piece. The diagnosis was made postmortem once the patient died after fast and progressive deterioration in the postoperative period.
\end{abstract}

\section{Introduction}

Aspergillosis is the disease caused by species of Aspergillus spp. These are ubiquitous in nature and their inhalation is common in the general population. However, tissue invasion and consequent infection are uncommon and usually involves some degree of immunosuppression, which alters the immune response to inhaled species. We present the case of a 71-year-old woman with a history of human immunodeficiency virus infection who was diagnosed postmortem with intestinal aspergillosis after histological examination of a segmental enterectomy piece.

\section{Case Description}

A 71-year-old woman with a past medical history of uterine cancer 25 years before, herpes-zoster infection two years before, recent diagnosis of human immunodeficiency virus (HIV) infection, and cervical adenopathies under investigation presented at the first medical appointment at the Infectious Diseases Unit referring a 3-week history of fever, weight loss of $20 \mathrm{~kg}$, and hemoptoic cough, as well as diarrhea with one year of evolution. On physical examination, she was cachectic and weak, had axillary temperature of $38^{\circ} \mathrm{C}$, blood pressure of $112 / 80 \mathrm{mmHg}$, respiration rate of 40 per minute, heart rate of 142 beats per minute, and oxygen saturation of $95 \%$ in room air. She also presented with pain and tenderness at the palpation of the hypogastric region, and during the consultation, she presented cardiorespiratory arrest. Advanced life support with favorable response was performed, and she was subsequently transferred to the emergency room where it was necessary to initiate aminergic support and proceed to orotracheal intubation and mechanical invasive ventilation. The complementary diagnostic exams revealed white blood cell count $14,740 / \mu \mathrm{L}$ with absolute neutrophil count $13,180 / \mu \mathrm{L}(89.4 \%)$ and absolute lymphocyte count $970 / \mu \mathrm{L}(6.6 \%)$ with $113 \mathrm{CD} 4+/ \mu \mathrm{L}$ cells, hemoglobin level $11.3 \mathrm{~g} / \mathrm{d}$, and platelet count $2,33,000 / \mu \mathrm{L}$. She presented with blood creatinine $1.34 \mathrm{mg} / \mathrm{dL}$, pancreatic amylase $222 \mathrm{U} / \mathrm{L}$ (4 times above the upper limit of normal), pancreatic lipase $174 \mathrm{U} / \mathrm{L}$ ( 3 times above the upper limit of normal), and seric lactates $6.5 \mathrm{mmol} / \mathrm{L}$. Viral load of HIV by polymerase chain reaction was 2,330,220 copies $/ \mathrm{mL}$. Thoracic, abdominal, and pelvic computed tomography (CT) revealed pneumoperitoneum, peritonitis, diffuse parietal thickening, and dilatation of the intestinal loops of the jejunum with splenic infarction (Figure 1).

An emerging surgery exploratory laparotomy was performed having been found enteric peritonites of the large cavity, occlusion with transition point at the level of the distal jejunum and poor perfusion, and thickening of the distal loops and perforation at the level of the distal 


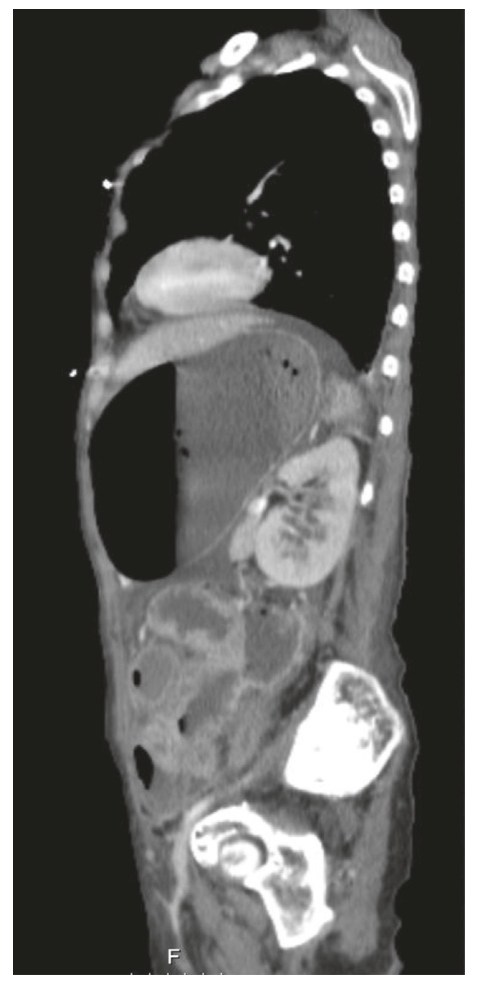

(a)

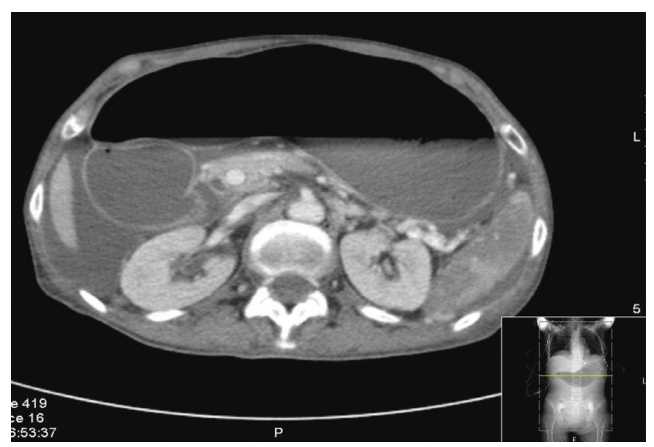

(b)

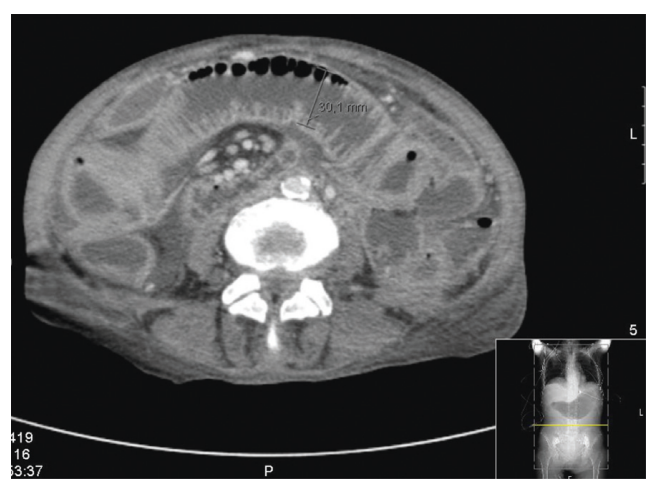

(c)

Figure 1: Thoracic, abdominal, and pelvic CT from the day of admission in (a) sagittal and (b, c) transverse planes.

ileum. Segmental enterectomy of approximately $35 \mathrm{~cm}$ of jejunum/ileum has been performed, including ischemic loop and ileal perforation. In the postoperative period, she was admitted to the intensive care unit, and broad-spectrum antibiotic therapy has been initiated. Nevertheless, she evolved with progressive clinical deterioration with increasing need of amines and without favorable hemodynamic response. The patient passed away the same day. The histological examination of the segmental enterectomy piece and available postmortem revealed acute transmural inflammation of the enteric wall, with suppuration and necrosis and extensive lesions of acute fibrinoexudative serositis with fungal hyphae of the Aspergillus spp. type. There were no images of angioinvasion, epithelioid granulomas, or signs of malignancy. The culture of peritoneal fluid obtained during surgery revealed polybacterial and fungal overinfection with Enterococcus faecium, Escherichia coli, and Candida albicans.

\section{Discussion}

Aspergillosis may refer to several types of diseases such as allergic disease, invasion of the upper or lower airways, skin infection, or extrapulmonary disease, which may be due to tissue invasion and hematogenous dissemination, which can lead to disseminated infection. Invasive aspergillosis (IA) occurs almost exclusively in situations where cellular immunity is severely compromised. In fact, when immunity is intact, Aspergillus conidia and hyphae are inhaled but destroyed by alveolar macrophages and polymorphonuclear leukocytes. When there is a qualitative or quantitative defect of these cells, there may be angioinvasive growth with hemorrhage, thrombosis, hypoxia, necrosis, and dissemination [1]. The majority of cases of IA are described in patients submitted to allogeneic hematopoietic cell transplant or solid organ transplant, those with severe and prolonged neutropenia, and those in need of high doses of glucocorticoids or immunosuppressive regimens [2]. HIV infection, although infrequent, is one of the risk factors for IA, being present in approximately $4 \%$ of the cases of IA described [2], even though IA occurs in less than $1 \%$ of HIV-infected patients [3, 4].

In this group of patients, IA has a high morbidity and mortality, with fatality rates around $86 \%[2,5]$. The risk of occurrence of IA increases with highest degree of immunosuppression or in those with other risk factors for invasive disease, such as neutropenia or use of corticoids [6]. The incidence is higher in patients with CD4+ cells below $100 / \mu \mathrm{L}$ and with one or more opportunistic diseases (acquired immunodeficiency syndrome (AIDS)-defining) [6]. However, in the highly active antiretroviral therapy (HAART) era, this paradigm has changed, with more cases being identified in patients with CD4+ cells higher then $100 / \mu \mathrm{L}$ [7]. It should be noted that in the present case, none of the other risk factors presented above were present, with the exception of low CD4+ cells.

Most cases of invasive aspergillosis occur in the lungs and respiratory tract, and patients with HIV infection are no exception (approximately $61.2 \%$ of the cases). Cases of intestinal IA described in the literature are extremely rare, constituting only isolated clinical cases. Although other routes of entry to IA other than pulmonary are only speculative, it is 
thought that in the intestine, the invasion may be through local inoculation and there are, as in the case of the patient presented, some cases described in patients with proven intestinal IA but without pulmonary disease [8-10]. Intestinal aspergillosis may manifest as enterocolitis, appendicitis, colonic ulcers, abdominal pain, and gastrointestinal bleeding.

The diagnosis of IA is considered "proven" when the hyphae of Aspergillus spp. are observed together with evidence of tissue injury (as presented in this case), or when a positive culture for Aspergillus spp. is obtained from a specimen obtained by sterile procedure and from a normally sterile site [11]. Whenever possible, microbiological cultures and histology should be obtained; however, isolated positive cultures may only be related to contamination of the samples.

\section{Conclusion}

Although very rare, patients with HIV infection/AIDS are susceptible to Aspergillus infection, which can lead to disseminated disease with high rates of morbidity and mortality, with most cases of extrapulmonary infections being documented postmortem. This case emphasizes the importance of early consideration of this differential diagnosis when approaching HIV-infected patients with symptoms similar to those of the patient under discussion.

\section{Conflicts of Interest}

The authors declare that they have no conflicts of interest.

\section{References}

[1] R. Ben-Ami, R. E. Lewis, and D. P. Kontoyiannis, "Enemy of the (immunosuppressed) state: an update on the pathogenesis of Aspergillus fumigatus infection," British Journal of Haematology, vol. 150, no. 4, pp. 406-417, 2010.

[2] S. J. Lin, J. Schranz, and S. M. Teutsch, "Aspergillosis casefatality rate: systematic review of the literature," Clinical Infectious Diseases, vol. 32, no. 3, pp. 358-366, 2001.

[3] K. B. Tong, C. J. Lau, K. Murtagh, A. J. Layton, and R. Seifeldin, "The economic impact of aspergillosis: analysis of hospital expenditures across patient subgroups," International Journal of Infectious Diseases, vol. 13, no. 1, pp. 24-36, 2009.

[4] M. Cornet, L. Fleury, C. Maslo, J.-F. Bernard, and G. Brücker, "Epidemiology of invasive aspergillosis in France: a six-year multicentric survey in the Greater Paris area," Journal of Hospital Infection, vol. 51, pp. 288-296, 2002.

[5] E. Mylonakis, T. F. Barlam, T. Flanigan, and J. D. Rich, "Pulmonary Aspergillosis and invasive disease in AIDS: review of 342 cases," Chest, vol. 114, no. 1, pp. 251-262, 1998.

[6] K. J. Holding, M. S. Dworkin, P. C. Wan et al., "Aspergillosis among people infected with human immunodeficiency virus: incidence and survival. Adult and Adolescent Spectrum of HIV Disease Project," Clinical Infectious Diseases, vol. 31, no. 5, pp. 1253-1257, 2000.

[7] B. Denis, M. Guiguet, N. de Castro et al., "Relevance of EORTC criteria for the diagnosis of invasive aspergillosis in hiv-infected patients, and survival trends over a 20 -year period in France," Clinical Infectious Diseases, vol. 61, no. 8, pp. 1273-1280, 2015.
[8] P. Eggimann, J. C. Chevrolet, M. Starobinski et al., "Primary invasive aspergillosis of the digestive tract: report of two cases and review of the literature," Infection, vol. 34, no. 6, pp. 333-338, 2006.

[9] M. González-Vicent, M. A. Díaz, I. Colmenero, J. Sevilla, and L. Madero, "Primary gastrointestinal aspergillosis after autologous peripheral blood progenitor cell transplantation: an unusual presentation of invasive aspergillosis," Transplant Infectious Disease, vol. 10, no. 3, pp. 193-196, 2008.

[10] D. P. Kontoyiannis, M. Mathur, Y. B. Chen et al., "Case records of the Massachusetts General Hospital. Case 13-2014. A 41-year-old man with fever and abdominal pain after stem-cell transplantation," New England Journal of Medicine, vol. 370, no. 17, pp. 1637-1646, 2014.

[11] B. De Pauw, T. J. Walsh, J. P. Donnelly et al., "Revised definitions of invasive fungal disease from the European Organization for Research and Treatment of Cancer/Invasive Fungal Infections Cooperative Group and the National Institute of Allergy and Infectious Diseases Mycoses Study Group (EORTC/MSG) Consensus Group," Clinical Infectious Diseases, vol. 46, no. 2, pp. 1813-1821, 1813. 


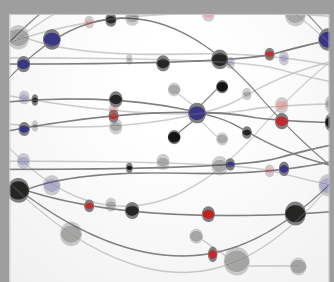

The Scientific World Journal
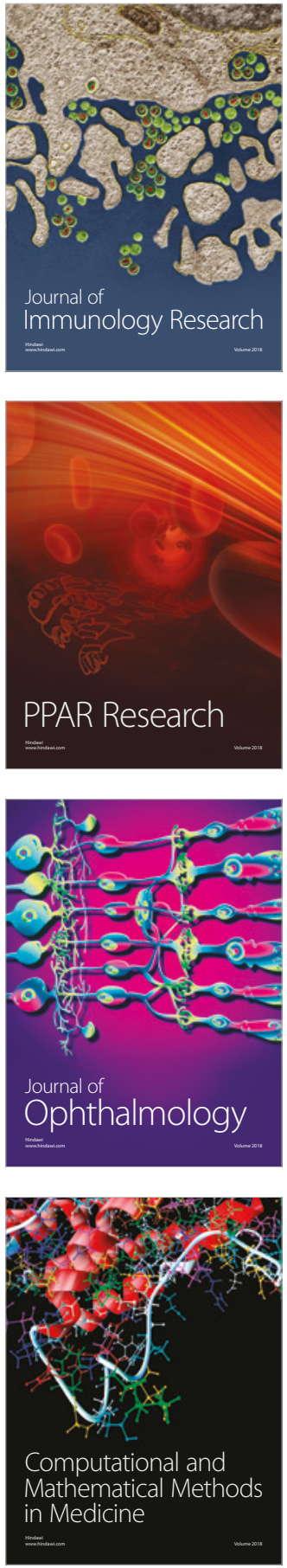

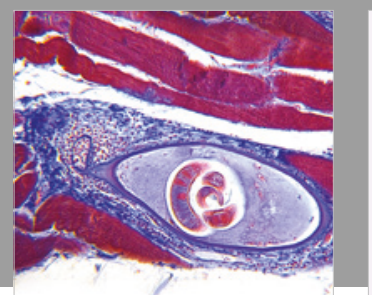

Gastroenterology Research and Practice

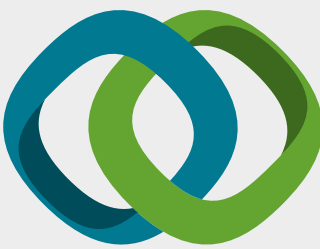

\section{Hindawi}

Submit your manuscripts at

www.hindawi.com
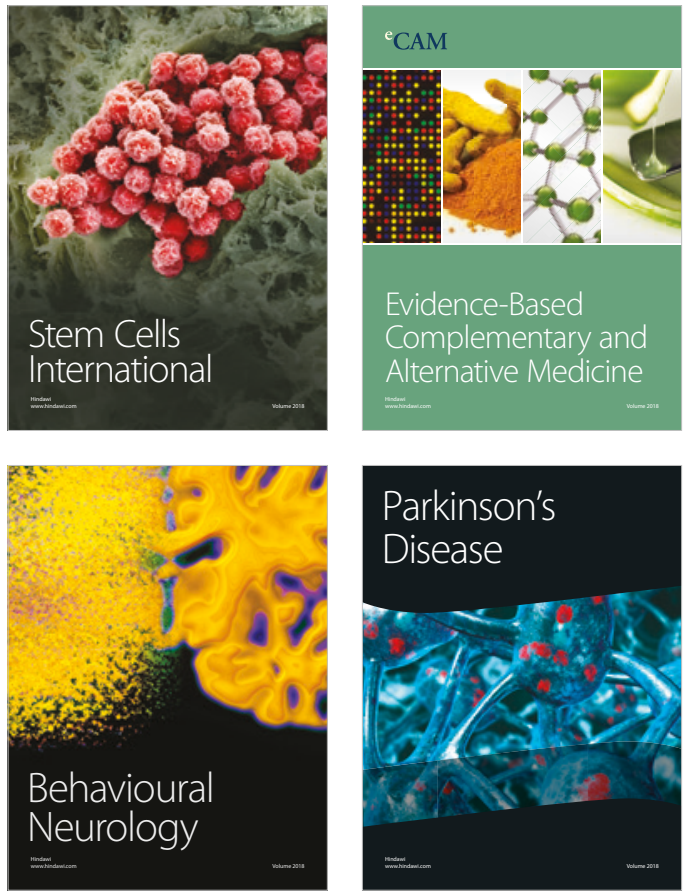

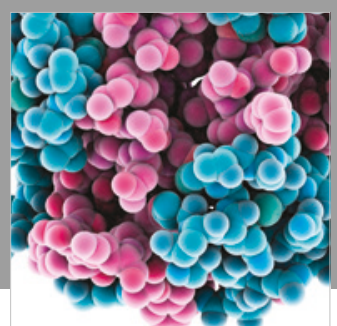

ournal of

Diabetes Research

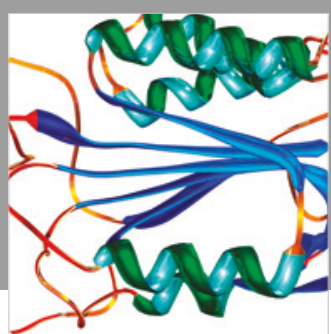

Disease Markers
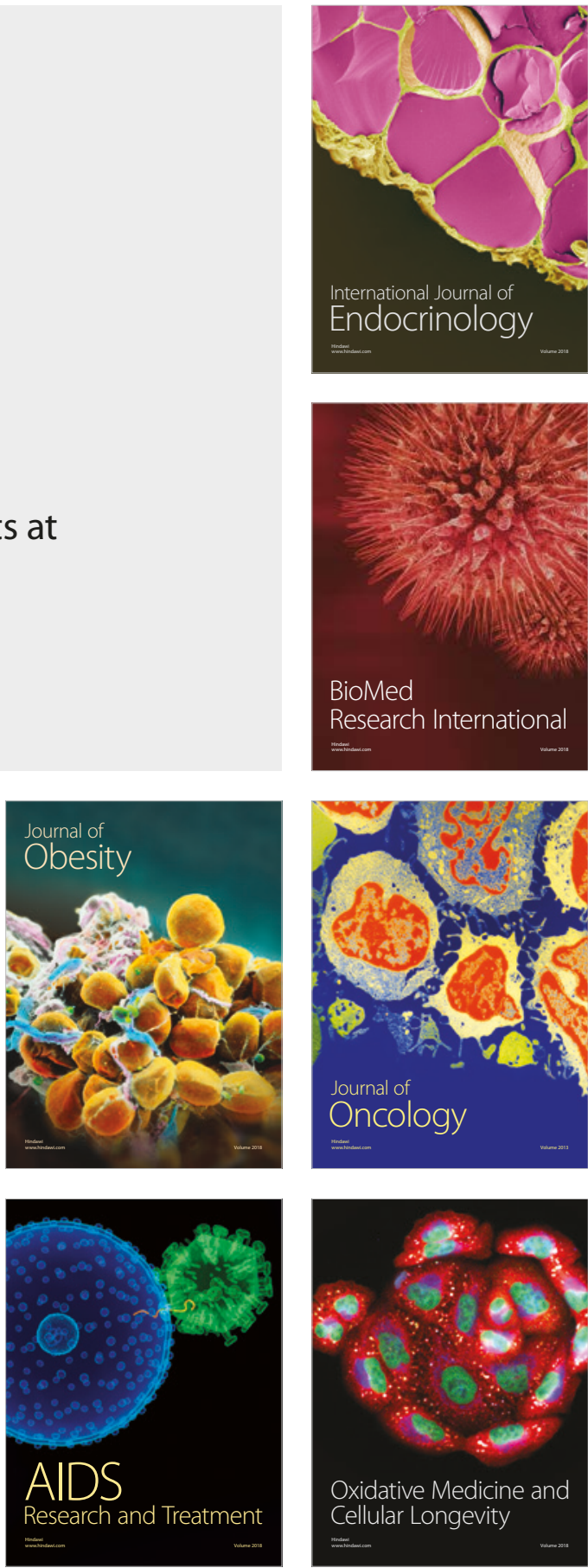